\title{
Macroscopic Findings Evaluator
}

National Cancer Institute

\section{Source}

National Cancer Institute. Macroscopic Findings Evaluator. NCI Thesaurus. Code C119859.

A person who determines the significance of a macroscopic findings assessment. 